\title{
Vibrotactile Sensitivity in Active Touch: Effect of Pressing Force
}

\author{
Stefano Papetti, Hanna Järveläinen, Bruno L. Giordano, Sébastien Schiesser, and Martin Fröhlich
}

\begin{abstract}
An experiment was conducted to study the effects of force produced by active touch on vibrotactile perceptual thresholds. The task consisted in pressing the fingertip against a flat rigid surface that provided either sinusoidal or broadband vibration. Three force levels were considered, ranging from light touch to hard press. Finger contact areas were measured during the experiment, showing positive correlation with the respective applied forces. Significant effects on thresholds were found for vibration type and force level. Moreover, possibly due to the concurrent effect of large (unconstrained) finger contact areas, active pressing forces, and long duration stimuli, the measured perceptual thresholds are considerably lower than what previously reported in the literature.
\end{abstract}

Index Terms—Vibrotactile thresholds, cutaneous sensitivity, touch psychophysics, finger, pressing force, contact area

\section{INTRODUCTION}

$\mathrm{T}$ HE psychophysics of active touch is still largely unknown, while most of the existing studies addressing touch consider it only as a receptive sense. Nevertheless, our everyday experience shows evidence that, e.g., manipulation and tactile exploration rely on active touch, and a better understanding of its underlying mechanisms would be highly relevant to haptics and human-computer interaction. As Gibson already observed in [1], "passive touch involves only the excitation of receptors in the skin and its underlying tissue", while "active touch involves the concomitant excitation of receptors in the joints and tendons along with new and changing patterns in the skin". Moreover, while passive touch is simply related to cutaneous sensitivity, active touch involves motor activity and cognitive processes that are likely to have an effect on tactile perception.

Cutaneous sensitivity has been generally studied in controlled passive settings, by considering frequency as the main independent variable and by observing sinusoidal stimuli. That led to identifying four channels in the skin which mediate different aspects of touch [2]: Vibrotactile perception is mainly conveyed by the non-Pacinian channel I (NP I)-sensitive to low-frequency vibrations in the $10-100 \mathrm{~Hz}$ range - and the Pacinian channel (P)—which shows a U-shaped contour of sensitivity in the $40-800 \mathrm{~Hz}$ band, with lowest thresholds between 200 and $300 \mathrm{~Hz}$.

A number of factors have been found to affect vibrotactile sensitivity at the hand, such as static force [3], [4], [5], [6], [7],

- S. Papetti, H. Järveläinen, S. Schiesser, and M. Fröhlich are with the Institute for Computer Music and Sound Technology, Zurich University of the Arts, Zurich 8005, Switzerland. E-mail: \{stefano.papetti, hanna. jarvelainen, sebastien.schiesser, martin.froehlich\}@zhdk.ch.

- B.L. Giordano is with the Institute of Neuroscience and Psychology, University of Glasgow, Glasgow G12 8QQ, United Kingdom.

E-mail:bruno.giordano@glasgow.ac.uk.

Manuscript received 20 Apr. 2016; accepted 29 May 2016. Date of publication 30 June 2016; date of current version 16 Mar. 2017.

Recommended for acceptance by H.Z. Tan.

For information on obtaining reprints of this article, please send e-mail to: reprints@ieee.org, and reference the Digital Object Identifier below.

Digital Object Identifier no. 10.1109/TOH.2016.2582485 contact area [3], [8], presence of a surround [4], [6], [8], temperature [2] stimulus duration [9], [10], skin indentation [11] and movement [12]. Although such factors are often interrelated (e.g., a surround also influences skin indentation and stiffness, and may results in static contact force), previous studies have shown that vibrotactile thresholds mediated by the Pacinian channel are typically lowered i) by increasing the static contact force, ii) by increasing the contact area (i.e., the Pacinian channel is sensitive to spatial summation), and iii) by increasing the duration of the stimuli (i.e., the Pacinian channel is sensitive to temporal summation).

However, experiments investigating the effect of passive static force on vibrotactile thresholds (e.g., [4], [5]) usually considered smaller forces as compared to those that may occur in active manual tasks.

A notable experiment described in [7] studied vibrotactile perceptual thresholds under active touch conditions, reporting thresholds much lower than in previous studies, except those reported in [13] for an experiment adopting similar procedures.

Vibrotactile sensitivity of hearing-impaired individuals was investigated in [14] for full open-hand contact, with spectrally and temporally complex signals: Thresholds were found considerably lower than elsewhere in the literature, and detection was reported for frequencies above those usually regarded as compatible with vibrotactile perception.

In a previous experiment by some of the present authors [15], vibration detection was studied on a piano keyboard during an active playing task: It was found that clearly perceivable vibrotactile cues are produced in the lower range of a piano keyboard, extending up to about the middle octave. However, as reported already by [16], vibration spectral peaks measured at these areas of the keyboard are generally well below the reference thresholds for passive touch [17], even for high dynamics playing styles.

The enhanced perception of vibration reported in the above studies may be explained by several concurrent factors, such as the presence of large contact areas (whole hand or finger pad), long stimulation times, and active touch. 
Notably, these seldom investigated stimulation conditions characterize many everyday tactual interactions, and potentially contribute to several attributes of the tactile percept, such as vibration intensity and frequency, or surface roughness and compliance. For these reasons, we suggest that the measurement of tactile perception under such conditions is highly relevant to the design and implementation of cutaneous feedback in human-computer interfaces. Indeed, novel technology and approaches have been developed in recent years, which open new possibilities in this regard [18], [19], [20]. Extending a previous study by some of the present authors [21], we designed an experiment to investigate vibrotactile sensitivity while addressing the following factors: active pressing force, unconstrained contact of the finger pad, broadband or sinusoidal vibration stimuli. In this rather ecological setting, sensitivity thresholds were examined as participants actively exerted different contact forces.

Enhanced tactile sensitivity has been previously observed in musicians compared to non-musicians [22] and, for instance, pianists' superior tactile performance has been linked directly to the duration of their daily practice [23]. Perceptual learning caused by short-term passive co-activation training produced a temporary enhancement in human tactile sensitivity, lasting for several hours after the training had finished [24], whereas the effects of long-term musical instrument training on tactile sensitivity are believed to be long-lasting [23]. Although differences between musicians and non-musicians are not the main objective of the present study, we looked for a similar effect in vibrotactile sensitivity. The experiment was therefore performed by both musicians and non-musicians.

\section{EXPERIMENT}

Vibrotactile perceptual thresholds at the finger were measured for different actively exerted pressing forces. Based on the results reported in [3], [4], [5], [6], [7], we expected perceptual thresholds to be influenced by the strength of the pressing force.

\subsection{Apparatus}

A tabletop device-shown in Fig. 1 and referred to as "touch-box" in what follows-was designed for the purpose of the experiment. Making use of a CZL635 load cell, the device is capable of measuring normal forces up to $49 \mathrm{~N}$ applied to its top surface. An Arduino UNO data acquisition board receives the analog force signal and samples uniformly at $1,920 \mathrm{~Hz}$ with 10-bit resolution [25]. Ad-hoc software developed in the Pure Data ${ }^{1}$ environment and running on a host computer receives the digital force data so produced and use them to synthesize vibrotactile stimuli (Section 2.2).

The stimuli are routed as audio signals through a RME Fireface 800 audio interface feeding a Kemo 031N audio amplifier connected to a TactileLabs Haptuator mark II, which is mounted perpendicularly at the lower side of the touch-box top panel. In addition, the device measures the area of contact of a finger touching its top surface. Similar to the technological solution described in [26], a strip of

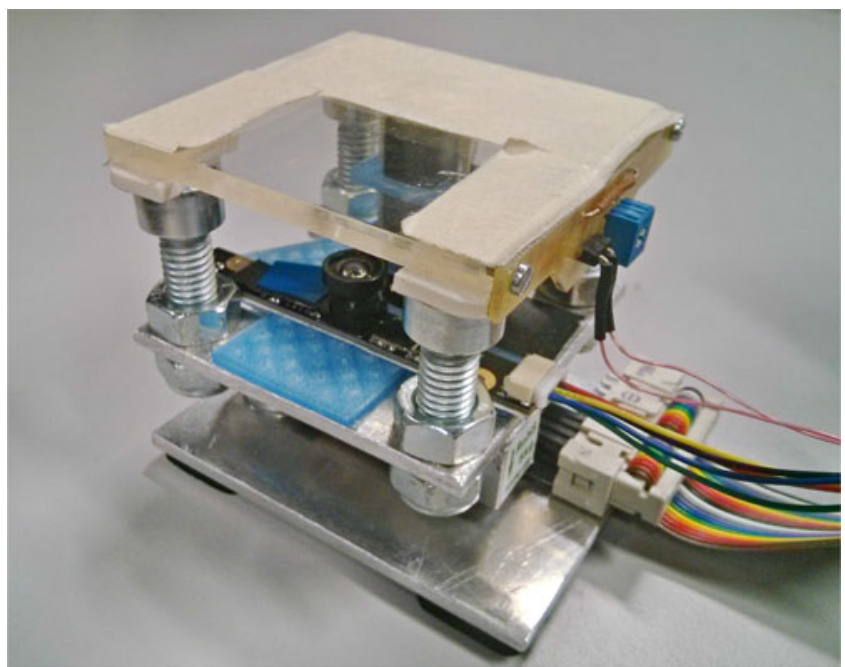

Fig. 1. The touch-box: The interface used in the experiment to yield vibrotactile feedback and to measure finger force and contact area.

infrared LEDs was attached at one side of the top panel, which is made of transparent Plexiglas: In this way, a finger pad touching the surface is illuminated by the infrared light passing through it. A miniature infrared camera placed under the top panel captures high-resolution $(1,280 \times 960$ pixels) images at $30 \mathrm{fps}$, and sends them via USB to a video processing software developed in the Max/Jitter environment, ${ }^{2}$ where the finger contact area is estimated.

Further details on contact force and area acquisition are given in the appendix, which can be found on the Computer Society Digital Library at http://doi.ieeecomputersociety. org/10.1109/TOH.2016.2582485.

The acoustical emissions originating from the vibromechanical actuator are minimal but perceivable. To eliminate their influence on the psychophysical results, during the experiment participants were presented with a masking noise through headphones. Also, the touch-box was placed on top of a stiff and thick rubber layer to forbid the propagation of vibration to the supporting table.

In order to minimize hand posture variability across participants, they were required to rest their forearm on a support while pressing their finger pad on top of the touch-box.

Finally, an LCD screen provided visual feedback on the correctness of the applied force and progress of the current trial.

\subsection{Stimuli and Conditions}

Two stimuli were implemented, which consisted either in a band-passed white noise with $48 \mathrm{~dB} /$ octave cutoffs at 50 and $500 \mathrm{~Hz}$, or a sine wave at $250 \mathrm{~Hz}$. Both the spectral range of the noise stimulus and the frequency of the sinusoidal stimulus were chosen so as to optimize the perceptibility of the vibrotactile stimulus by focusing around the range $200-300 \mathrm{~Hz}$, known to results in peak sensitivity for passive touch [17].

The amplitude of the stimuli was varied in fixed steps according to a staircase procedure (Section 2.3). The initial amplitude was set after a pilot study, so that vibration at the first trial would be clearly detected by participants. 
In order not to exceed one hour of session duration, force levels were limited to three. They were chosen based on a pilot study and on what reported in [27], so as to cover a range from light touch to hard press while still comfortable for participants as well as suitable for application in human-computer interfaces. The three force levels are referred to as Low, Mid and High, and correspond respectively to $1.9,8$ and $15 \mathrm{~N}$, with a tolerance of $\pm 1.5 \mathrm{~N}$.

\subsection{Design and Task}

The experimental protocol followed the guidelines for nonclinical experiments involving human participants and was approved by the Cantonal Ethics Committee of Zurich.

Perceptual thresholds were measured using a one-uptwo-down staircase algorithm with fixed step size ( $2 \mathrm{~dB})$ and eight reversals, and a two-alternative forced choice (2AFC) procedure. The method estimates the stimulus level corresponding to a correct detection rate of 70.7 percent [28]. Three staircases-corresponding to the requested force levels-were interleaved so that each block of three trials contained one trial from each staircase, in random order.

Participants were seated at a table, the touch-box and LCD screen in front of them, and rested their forearm on the arm support. They were instructed to use their dominant index finger throughout the experiment, and not touch the box with other fingers. A trial consisted of observation intervals ' $A$ ' and ' $B$ ', with the stimulus randomly presented in only one of them. The task was to detect which interval contained the stimulus. Participants would press on the top panel of the touch-box with increasing force until the LCD screen turned green, signaling that the correct force level was reached. After the pressing force was kept stable over a $1 \mathrm{~s}$ stabilization interval, a $1.5 \mathrm{~s}$ observation interval followed and the procedure was repeated. Participants told their response to the experimenter, who recorded it into the experimental software. The experiment lasted between 35 and 60 minutes, depending on the participants' performance. A 1-minute break was allowed every 5 minutes.

Twenty-seven subjects participated in the sinusoidal condition, and seventeen in the noise condition. They where 1939 years old $($ mean $=26, \mathrm{sd}=4.5)$ and half of them were music students.

A previous experiment considering only the noise condition had been performed a year earlier without contact area measurement [21]. One participant in each condition of the experiment presented here had also performed the previous experiment. Since a new device was developed for the present study to measure both force and contact area, the noise condition was repeated. Only twelve of the participants in the sine condition took part in the new noise condition, which was performed 2-4 weeks later; the rest of the individuals in the noise condition were new. This led to unbalanced group sizes, and to a mixed experimental design with one withinsubjects factor (pressing force: low, middle or high) and one between-subjects factor (vibration profile: sine or noise). The possible effects of condition order and participants' varying familiarity to the task is addressed in Section 3.

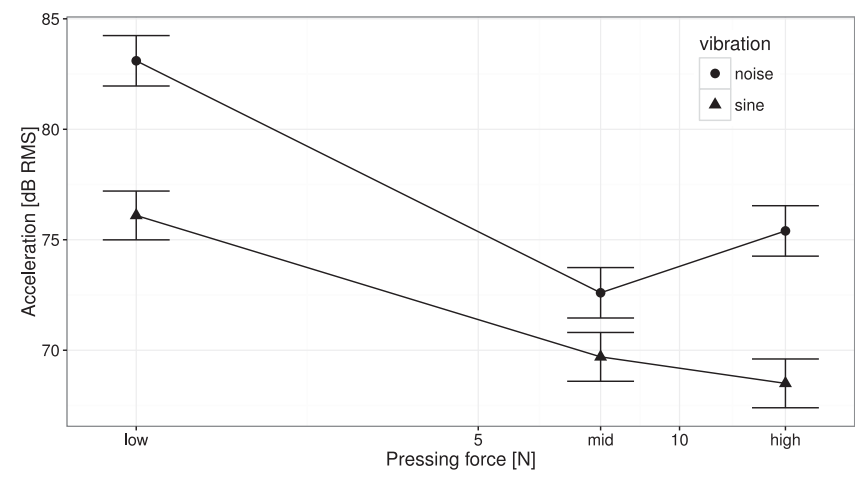

Fig. 2. Thresholds measured at three pressing force levels, for sinusoidal and noise vibrations. Error bars $\pm \mathrm{SE}$.

\subsection{Characterization}

Different experiments investigating touch psychophysics may provide divergent results due to the varying accuracy of haptic devices [29]. For this reason, we suggest that procedures for validation and characterization of experimental devices should be adopted in touch psychophysics studies. The characterization of vibration output is especially relevant to studies on vibrotactile sensitivity, as it allows one to compare the actual stimuli as delivered to the skin with the original stimuli feeding the experimental device.

Before performing the experiment, the accuracy of our equipment was measured and analyzed in order to calibrate force and contact area sensing and to characterize the produced vibration. Details are provided in the appendix, available in the online supplemental material of this paper, while only the most relevant findings are reported here. While a consistent behavior was measured in the amplitude response of both sinusoidal and noise vibration, the vibration spectrum in the noise condition was affected by pressing force. More specifically, the spectral centroid (roughly representing the 'center of mass' of a spectrum) of noise vibration shifted from about $180 \mathrm{~Hz}$ in the Low force condition to up to about $380 \mathrm{~Hz}$ in the Mid and High conditions. Also, the spectral centroid was generally found to decrease slightly with vibration amplitude. How this may have influenced the results is addressed in Section 4.

\section{REsults}

Statistical analyses were carried out using $\mathrm{R}$ and the packages afex, lsmeans, and Rfit. $^{3}$

\subsection{Effect of Pressing Force on Vibrotactile Thresholds}

Vibration perception thresholds were calculated for each participant and each pressing force condition as the mean of the stimulus level during the last six reversals of the staircase (arithmetic mean of rms acceleration values, shown in Fig. 2 and Table 1).

For each pressing force level, thresholds for sinusoidal vibration were lower than for noise. For both vibration conditions, higher thresholds (i.e., worse detection performance) were obtained at the Low force condition, while at the other two force levels the thresholds were generally

3. http://www.R-project.org/ 
TABLE 1

Mean Thresholds Expressed as Rms Acceleration and Respective Standard Deviation in Brackets $\left(\mathrm{dB}\right.$, re $\left.10^{-6} \mathrm{~m} / \mathrm{s}^{2}\right)$

\begin{tabular}{lcccc}
\hline & \multicolumn{2}{c}{$\begin{array}{c}\text { Sinusoidal condition } \\
(\mathbf{N}=\mathbf{2 7})\end{array}$} & & $\begin{array}{c}\text { Noise condition } \\
(\mathrm{N}=17)\end{array}$ \\
\cline { 2 - 3 } \cline { 5 - 5 } & $\begin{array}{c}\text { Acceleration } \\
(\mathrm{rms})\end{array}$ & $\begin{array}{c}\text { Displacement } \\
(\text { peak })\end{array}$ & & $\begin{array}{c}\text { Acceleration } \\
(\mathrm{rms})\end{array}$ \\
\hline Low & $76.1(6.1) \mathrm{dB}$ & $-48.7 \mathrm{~dB}$ & & $83.1(5.2) \mathrm{dB}$ \\
Mid & $69.7(5.3) \mathrm{dB}$ & $-58.2 \mathrm{~dB}$ & & $72.6(5.3) \mathrm{dB}$ \\
High & $68.5(4.0) \mathrm{dB}$ & $-59.3 \mathrm{~dB}$ & & $75.4(5.5) \mathrm{dB}$ \\
\hline
\end{tabular}

Corresponding peak displacement calculated for the sinusoidal condition $(\mathrm{dB}$, re $\left.10^{-6} \mathrm{~m}\right)$.

lower: The lowest mean threshold ( $68.5 \mathrm{~dB}$ rms acceleration) was measured at the High force condition with sinusoidal vibration, and the highest at the Low force condition with noise vibration $(83.1 \mathrm{~dB})$ - thus thresholds vary over a wide range across conditions. Individual differences were also large: The lowest and highest individual thresholds differ typically by about $20 \mathrm{~dB}$ in each condition.

Perceptual thresholds were analyzed by means of a 2 between-subject (type of vibration: [sinusoidal; noise]) $\times 2$ between-subject (musical experience: [musician; non-musician]) $\times 3$ within-subject (pressing force: [Low; Mid; High]) repeated measures ANOVA. The sphericity assumption was not violated (Mauchly's $W=0.98, p=0.64$ ). There was a significant main effect of type of vibration $\left(F_{1,41}=14.64\right.$, $p<0.001$, generalized $\left.\eta^{2}=0.23\right)$ and of force level $\left(F_{2,82}=137.5, p<0.0001, \eta^{2}=0.35\right)$, while the main effect of musical experience was not significant $\left(F_{1,41}=0.36\right.$, $p>0.05)$. Significant interactions were observed between type of vibration and force $\left(F_{2,82}=8.41, p<0.001\right)$, and musical experience and force $\left(F_{2,82}=6.64, p=0.002\right)$. In the Low force condition, musicians' mean thresholds were about $3 \mathrm{~dB}$ lower than those of non-musicians, while the difference remained within $1 \mathrm{~dB}$ for the other two force levels. However the respective effect size was very small $\left(\eta^{2}=0.03\right)$.

Post-hoc pairwise comparisons with Bonferroni correction indicated that the Low force condition differed from both the Mid and High force conditions, for both vibration types: Comparison with Mid force resulted in $t(82)=12.0$, $p<0.0001$ for noise and $t(82)=9.02, p<0.0001$ for sinusoidal vibration; comparison with High force resulted in $t(82)=8.85, p<0.0001$ for noise and $t(82)=10.6, p<$ 0.0001 for sinusoidal vibration. For noise vibration, the difference between Mid and High force conditions was significant $(t(82)=-3.17, p=0.02)$, but the respective contrast for sinusoidal vibration was not $(t(82)=1.64, p>0.05)$. The difference between sinusoidal and noise vibrations was significant for the Low $(t(57.44)=4.37, p<0.001)$ and High $(t(57.44)=4.29, p<0.001)$ force conditions, but not for the Mid force $(t(57.44)=1.85, p>0.05)$.

Two participants-one in each vibration condition-had taken part in a related experiment a year earlier [21]. In order to verify the possible effect of learning on them, t-tests were computed to compare their individual mean thresholds in the current experiment with those of the remaining naive participants. No significant evidence of a worse performance in naive versus "experienced" individuals was
TABLE 2

Mean Pressing Forces and Respective Standard Deviation in Brackets

\begin{tabular}{lcc}
\hline & w/ vibration & w/o vibration \\
\hline & \multicolumn{2}{c}{ Sinusoidal condition $(\mathrm{N}=27)$} \\
\cline { 2 - 3 } Low $(1.9 \mathrm{~N})$ & $1.54(0.40) \mathrm{N}$ & $1.49(0.41) \mathrm{N}$ \\
Mid $(8 \mathrm{~N})$ & $7.62(0.25) \mathrm{N}$ & $7.58(0.26) \mathrm{N}$ \\
High $(15 \mathrm{~N})$ & $14.4(0.16) \mathrm{N}$ & $14.4(0.17) \mathrm{N}$ \\
& \multicolumn{2}{c}{ Noise condition $(\mathrm{N}=17)$} \\
\cline { 2 - 3 } Low $(1.9 \mathrm{~N})$ & $1.45(0.47) \mathrm{N}$ & $1.49(0.49) \mathrm{N}$ \\
Mid $(8 \mathrm{~N})$ & $7.62(0.32) \mathrm{N}$ & $7.67(0.29) \mathrm{N}$ \\
High $(15 \mathrm{~N})$ & $14.4(0.20) \mathrm{N}$ & $14.4(0.21) \mathrm{N}$ \\
\hline
\end{tabular}

found: In the sine condition, naive participants perform'ed, on average, better than the experienced participant at the Low force level $(t(25)=-6.33, p<0.0001)$, while at the Mid and High force levels, the experienced individual did not perform differently than the naive participants $(t(25)=0.88$ and $t(25)=1.48, p \geq 0.14)$. In the noise condition, experienced participants did not perform differently than the naive participants at all force levels $(t(10)=-1.22$, $t(10)=0.15$ and $t(10)=-0.54, p \geq 0.11)$.

Twelve individuals participated in both experimental conditions. Their data were further analyzed as specified above, with repeated measures on both type of vibration and pressing force, giving very similar results to what observed for the full dataset. These additional analyses are therefore not presented. However, similarity of the results regardless of having participated in only one or both conditions, as well as the fact that the noise condition-which was performed 2-4 weeks later-produced higher thresholds, suggest that familiarity with the task did not bring any significant benefit.

\subsection{Analysis of Pressing Force}

Mean force and its standard deviation were measured for each individual press. Pressing stability can be described as the mean of the standard deviations of each press, at each force level. Presence or type of vibrations had no significant effect on individual pressing stability: The mean standard deviations were typically 5,2 , and 1.5 percent of the mean pressing force, respectively for the Low, Mid and High conditions, regardless of vibrations. Mean overall pressing forces and respective standard deviations are presented in Table 2, showing that the presence or type of vibrations had no significant effect on them either.

Due to the fixed $1.5 \mathrm{~N}$ tolerance, pressing forces could go occasionally relatively far from the nominal $1.9 \mathrm{~N}$ in the Low force condition: The lowest participant-specific mean forces were $0.75 \mathrm{~N}$ in the sine condition and $0.87 \mathrm{~N}$ in the noise condition, however only one participant pressed on average softer than $1.0 \mathrm{~N}$ in the sine condition and three in the noise condition.

Participant-specific thresholds in the sinusoidal condition were negatively correlated with the mean pressing force (mean Pearson's $\rho=-0.85$ ). Fig. 3 presents individual thresholds in the sinusoidal condition as a function of force on a logarithmic scale. For about $2 / 3$ of the participants, thresholds decrease monotonously with increasing pressing force. In the noise condition, however, the mean correlation 


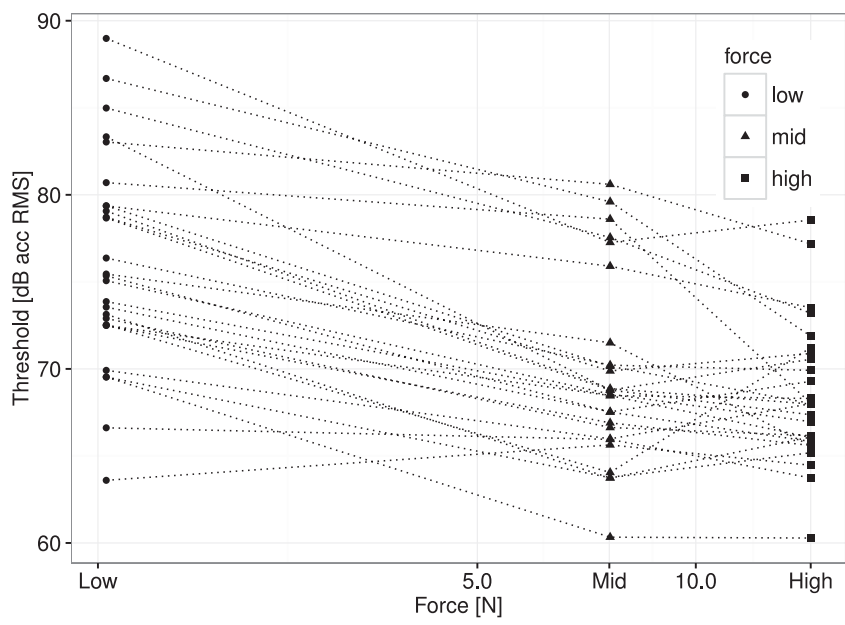

Fig. 3. Individual thresholds as a function of pressing force for each participant $(\mathrm{N}=27)$ in the sinusoidal vibration condition.

was lower (mean $\rho=-0.76$ ), and the results have an apparent U-shape, as seen in Fig. 2.

\subsection{Analysis of Contact Area}

Area data were measured throughout the sinusoidal condition $(\mathrm{N}=27)$ but, due to technical issues, area measurement was available for only six participants in the noise condition. Mean contact areas and the respective standard deviations are presented in Table 3. Since pressing style was not constrained, individual differences were high, resulting in contact areas varying across a range of about $100 \mathrm{~mm}^{2}$.

As shown in Fig. 4, mean contact area increased roughly linearly when representing pressing force on a logarithmic scale, that is an almost logarithmic relationship is found between area and force. Mean contact areas were slightly smaller in the noise condition than in the sinusoidal one, even though pressing forces were stable. Since area measurement was done on considerably different group sizes for the two vibration conditions, we studied more closely the six participants who participated in both conditions and had area data available: The mean areas were approximately 10 percent smaller in the noise condition, however the effect was not significant (ANOVA with repeated measures on vibration type and force: $F(1,5)=3.25, p>0.05)$.

As pressing force level and mean contact area were positively correlated (average participant-specific correlations: Pearson's $\rho=0.93$ for sinusoidal and $\rho=0.96$ for noise vibration), the relationship between area and thresholds was studied more closely. Fig. 5 presents individual thresholds in the sinusoidal condition as a function

TABLE 3

Mean Finger-Contact Area, and Respective Standard Deviation in Brackets

\begin{tabular}{lcc}
\hline & Sinusoidal vibration $(\mathbf{N}=\mathbf{2 7})$ & Noise vibration $(\mathbf{N}=\mathbf{6})$ \\
\hline Low & $117(35) \mathrm{mm}^{2}$ & $103(30) \mathrm{mm}^{2}$ \\
Mid & $163(39) \mathrm{mm}^{2}$ & $151(35) \mathrm{mm}^{2}$ \\
High & $175(39) \mathrm{mm}^{2}$ & $168(34) \mathrm{mm}^{2}$ \\
\hline
\end{tabular}

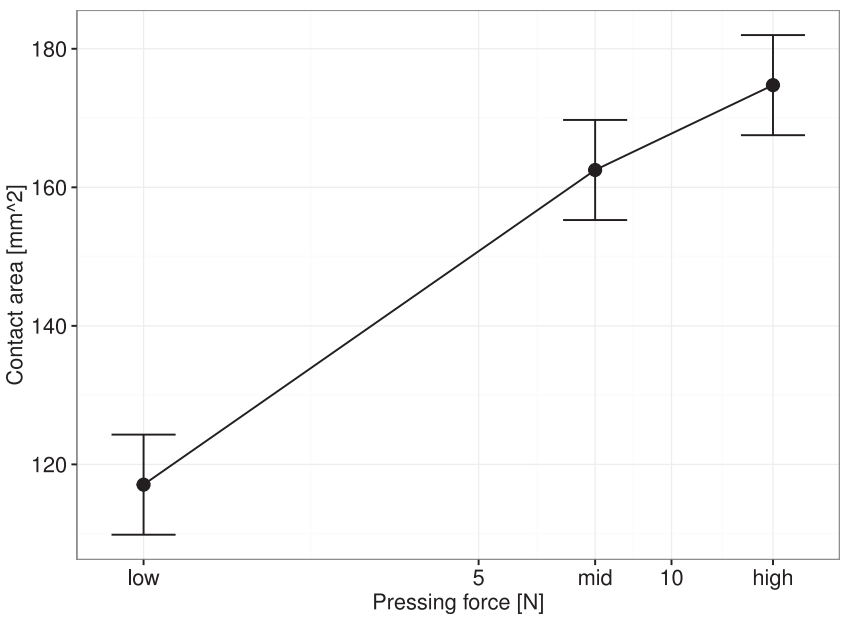

Fig. 4. Contact area as a function of pressing force in the sinusoidal vibration condition. Error bars $\pm \mathrm{SE}$.

of contact area: For about $2 / 3$ of the participants thresholds decreased monotonously with increasing contact area, while the remaining participants show a U-shaped trend. The mean correlation between participant-specific thresholds and participant-specific contact areas was $\rho=-0.82$ in the sinusoidal condition and $\rho=-0.88$ in the noise condition, although in the latter the trend was more clearly U-shaped. Thus the behavior of the thresholds seems similar in relation to both pressing force (on a logarithmic scale) and contact area.

Participants' sensitivity to vibrations changed with pressing force and contact area, but there were high individual differences in general level of sensitivity. As only pressing force was systematically controlled in the present experiment and there are few data points per participant (three force levels), it is currently impossible to say whether contact area or pressing force would better predict sensitivity to vibration at the participant-specific level. However we investigated the extent to which contact area explained across-participant variability. To this purpose, a new dependent variable was formed as the participant-specific threshold averaged across force levels, and a new independent

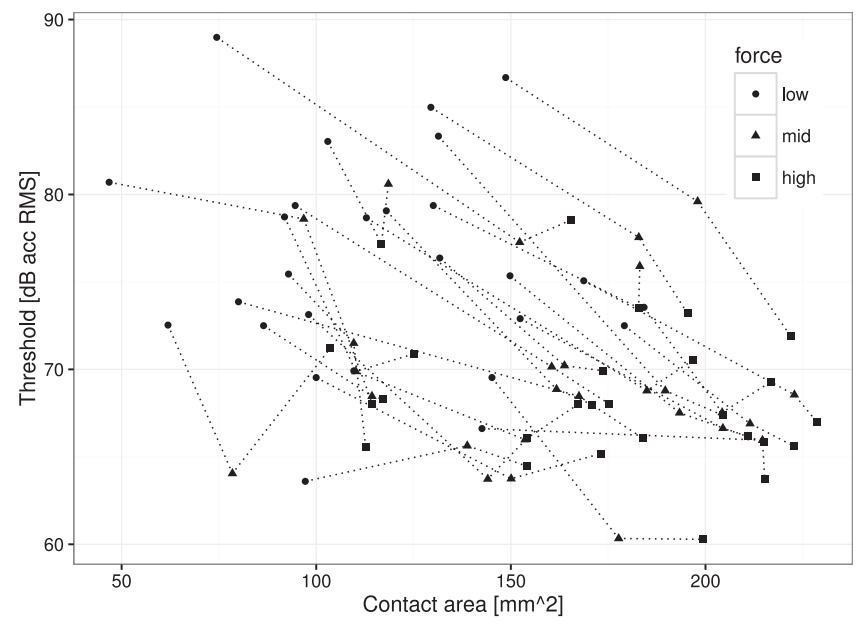

Fig. 5. Thresholds as a function of contact areas for individual participants $(\mathrm{N}=27)$ in the sinusoidal vibration condition. 
variable as the participant-specific area averaged across force levels. A multiple rank-regression model was computed using these variables, and age and musical experience as further explanatory variables: The resulting slope estimates, standard errors and p-values were respectively $-0.04,0.02, p=0.17$ for area, $-3.21,1.8, p=0.09$ for musical experience, and $0.25,0.2, p=0.24$ for age. Although the effect of musical experience approaches significance, none of the considered predictors appears to have a significant effect at the $p<0.05$ level. Whilst contact area is reliably associated with pressing force at the participantspecific level, it is not a significant predictor of interindividual differences in tactile sensitivity. Additional studies will be required to further isolate the contribution of contact area during active pressing tasks.

\section{Discussion}

\subsection{Interpretation of Results}

By using stimuli centered around the frequency range of $200-300 \mathrm{~Hz}$, the present study focused in the known area of peak sensitivity for passive touch [17], and investigated the effect of actively exerted forces on vibrotactile perception thresholds. A more general characterization is a vast task and would require adding stimuli of various nature and frequency range under both active and passive force exertion, as well as control of contact area. Such a large-scale study remains a future task.

Under the condition of active pressing force, vibrotactile perceptual thresholds were found ranging from 68.5 to $83.1 \mathrm{~dB}$ rms acceleration (re $10^{-6} \mathrm{~m} / \mathrm{s}^{2}$ ), values that are considerably lower than what generally reported in the literature. Maeda and Griffin [6] compared acceleration thresholds from various studies addressing passive touch, finding that most of them are in the range $105-115 \mathrm{~dB}$ for sinusoidal stimuli ranging from 100 to $250 \mathrm{~Hz}$. The lowest reported acceleration thresholds are $97-98.5 \mathrm{~dB}$, for contact areas (probe size) ranging from 53 to $176.7 \mathrm{~mm}^{2}$ [30], [31], [32]. It is worth noticing that the widely accepted results by Verrillo [17] report lowest displacement thresholds of approximately $-20 \mathrm{~dB}\left(\right.$ re $\left.10^{-6} \mathrm{~m}\right)$ at $250 \mathrm{~Hz}$, equivalent to about $105 \mathrm{~dB}$ rms acceleration. ${ }^{4}$

The main result of the present experiment is that vibrotactile sensitivity was found to depend on the applied pressing force. Thresholds were highest at the Low force condition, and decreased significantly at both Mid and High force levels. In good accordance with what reported in our preliminary study [21], for noise vibration the lowest threshold was obtained at the Mid force condition, while at the Low and High conditions thresholds were higher, resulting in a U-shaped threshold contour with respect to the applied force. However, considering that the spectral centroid of the noise vibration generally shifted towards $300 \mathrm{~Hz}$ and higher frequencies for the Mid and High force conditions (Section 2.4 and the appendix, available in the online supplemental material), we suggest that the U-shape of the threshold-force curve might be partially due to the

4. It is straightforward to convert between acceleration and displacement for sinusoidal vibration: acc $=\operatorname{displ} \cdot(2 \pi f)^{2}$, where $f$ is the frequency. Also, $\mathrm{x}_{\mathrm{rms}}=\mathrm{x}_{\mathrm{peak}} / \sqrt{2}$. response of the Pacinian channel, which has a U-shaped contour over the frequency range $40-800 \mathrm{~Hz}$ with maximum sensitivity in the 200-300 Hz range [2]. Conversely, for sinusoidal vibration at $250 \mathrm{~Hz}$, mean $\mathrm{dB}$ thresholds decreased roughly logarithmically for increasing pressing forces (see Fig. 2). This simpler trend may be due to the more consistent behavior of our system when reproducing simpler sinusoidal vibration. An improved version of the touch-box would be needed to test whether a similar trend can be found when noise stimuli are reproduced more linearly for varying pressing forces.

Further studies are needed to precisely assess how vibratory thresholds might be affected by passive forces of strength equivalent the active forces used in the present study. However, since the Low condition in our experiment was already satisfied by applying light pressing force (the measured mean is about $1.49 \mathrm{~N}$ ), we suggest that it may be compared to studies addressing passive static forces. Craig and Sherrick [3] found that increasing static force on the contactor produces an increase in vibrotactile magnitude. They considered vibration bursts at 20,80 and $250 \mathrm{~Hz}$ lasting $1240 \mathrm{~ms}$, contact areas up to $66.3 \mathrm{~mm}^{2}$, and static forces of about 0.12 and $1.2 \mathrm{~N}$. Lamoré and Keemink [4] report that, for a contact area of $1.5 \mathrm{~cm}^{2}$ and no surround, an increase in static force at the fingertip from 0.5 to $2 \mathrm{~N}$ increases the threshold at frequencies below $40 \mathrm{~Hz}$ and has no effect for frequencies above. Conversely, for $4.5 \mathrm{~N}$ static force at the forearm and thenar eminence, they found lower thresholds above $40 \mathrm{~Hz}$. Harada and Griffin [5] used a contact area of $38.5 \mathrm{~mm}^{2}$ and found that higher forces in the range 1-3 $\mathrm{N}$ led to significant lowering of thresholds by 2 to $6 \mathrm{~dB} \mathrm{rms}$ at 125, 250, and $500 \mathrm{~Hz}$. The lowest thresholds reported are however around $100 \mathrm{~dB}$ rms acceleration. Similar to the effect of static force, in [33] the fingertip was strained by blood constriction. As a result, skin stiffness increased, while the contact area was reduced (unlike our study, where higher pressing forces resulted in larger contact areas). Vibration sensitivity slightly improved with finger stiffness. On the other hand, Brisben et al. [7] reported that passive static contact forces from 0.05 to $1.0 \mathrm{~N}$ did not have an effect on thresholds. However, with only four participants, the statistics of those results are not robust. Nevertheless, the authors suggested that extending these investigations to higher forces-as found in everyday life-would be important. They also hypothesized that increasing the force beyond 1-2 N could lower thresholds by better coupling of vibrating surfaces to bones and tendons, which could result in more effective vibration transmission to distant Pacinian corpuscles. That might also help explaining the generally lower thresholds that we found for higher forces. In our study, force level was found strongly correlated to contact area, resulting in larger areas for higher forces, which clearly contributed to further lowering perceptual thresholds.

Only a few studies are found in the literature that deal with non-sinusoidal stimuli. Gescheider et al. [34] studied difference limens for the detection of changes in vibration amplitude, with either sinusoidal stimuli at 25 or $250 \mathrm{~Hz}$ or narrow-band noise with spectrum centered at $175 \mathrm{~Hz}$ and $24 \mathrm{~dB}$ /octave falloff at 150 and $200 \mathrm{~Hz}$ (contact area $2.9 \mathrm{~cm}^{2}$ ): 
They found that the nature of the stimuli had no effect on difference limens. Wells et al. [35] studied stochastic resonance due to the superimposition of a white noise upon sinusoidal stimuli, and reported enhanced detection at the foot soles of both elderly people with elevated vibrotactile thresholds and young people with normal thresholds. Wyse et al. [14] conducted a study with hearingimpaired participants and found that, for complex stimuli and whole hand contact (area of about $50-80 \mathrm{~cm}^{2}$ ), the threshold at $250 \mathrm{~Hz}$ was $80 \mathrm{~dB}$ rms acceleration (re $10^{-6} \mathrm{~m} / \mathrm{s}^{2}$ ), that is comparable with our results, especially in the low force condition. In that study, it is hypothesized that the temporal dynamics of spectrally complex vibration might play a key role in detecting vibrotactile stimulation. In our case, however, the stimuli had no temporal dynamics. In the present study sinusoidal stimuli resulted in lower rms acceleration thresholds as compared to noise vibration. Intuitively, this may be explained by considering that equivalent rms acceleration values for sinusoidal and noise stimuli actually result in a similar amount of vibration power ${ }^{5}$ being concentrated at $250 \mathrm{~Hz}$ (a frequency characterized by peak tactile sensitivity [17]), or spread across the $50-500 \mathrm{~Hz}$ band, respectively. This explanation is somehow confirmed by the findings reported in [36], where sinusoidal stimuli resulted in lower thresholds as compared to spectrally more complex signals (square and ramp waves).

The Pacinian channel, targeted by this study, is capable of spatial summation. In [17] and [37] it is shown that for contact areas between 2 and $510 \mathrm{~mm}^{2}$ at the thenar eminence of the hand, and for frequencies in the $40-800 \mathrm{~Hz}$ range, displacement thresholds decrease by approximately $3 \mathrm{~dB}$ with every doubling of the area. Intuitively, a reason for that is that the number of stimulated skin receptors increases with larger contact areas. In the present study, finger contact areas were measured during an active pressing task: Participants were required to reach and maintain target forces, while they were allowed to use a pressing style of their choice, resulting in high variability of the contact area. Under these conditions, a roughly logarithmic relationship was found between contact area and force (see Fig. 4), however the effect of area only on thresholds could not be isolated. The lowering of thresholds by $3 \mathrm{~dB}$ with every doubling of contact area, reported by Verrillo [17], refers on the other hand to a logarithmically decreasing trend. Our results cannot confirm this as-although the range of contact areas was small in our study compared to Verrillo'sareas were varying and were not systematically controlled. It remains a future task to isolate the effects of force and area in active pressing. However, even though the increase of contact area likely contributes to the lowering of thresholds with pressing force, taken alone it did not explain individual differences. Generally speaking, mean contact areas were in the range $103-175 \mathrm{~mm}^{2}$, contributing to explain the reported enhanced sensitivity.

5. Indeed, rms is the square root of averaged power in the considered time interval (stimulation time). Therefore comparing rms vibration is equivalent to comparing vibration power. Also, since the considered signals are stationary, their averaged power is the same as instantaneous power.
The Pacinian channel is also sensitive to temporal summation, which lowers sensitivity thresholds and enhances sensation magnitude [37]. Verrillo [9] found that thresholds decrease for stimuli at $250 \mathrm{~Hz}$ for increasing duration up to about $1 \mathrm{~s}$, when delivered through a $2.9 \mathrm{~cm}^{2}$ contactor to the thenar eminence of the hand. Gescheider and Joelson [10] examined temporal summation at stimulus intensities ranging from the threshold to $40 \mathrm{~dB}$ above it: For 80 and $200 \mathrm{~Hz}$ stimuli, peak displacement thresholds were lowered by up to about $8 \mathrm{~dB}$ for duration increasing from 30 to $1,000 \mathrm{~ms}$. The present study made use of $1.5 \mathrm{~s}$ stimuli, which likely contributed to enhance vibrotactile sensitivity.

Large interindividual differences in sensitivity were found in our experiment, which we could not fully explain by contact area or age. However, this observation is in accordance with, e.g., [6], [32], [38], [39], where large interand intraindividual differences were reported. Sources for large variations in sensitivity may be many. While exposure to vibration is a known occupational health issue and can cause acute impairment of tactile sensitivity [5], experience in conditions similar to the present experiment seemed a possible advantage. Therefore we further analyzed the performance of musician participants, who are often exposed to vibrations when performing on their instruments: Indeed, musicians' mean threshold in the Low force condition was about $3 \mathrm{~dB}$ lower than non-musicians', but there was no significant difference at the other force levels. Overall, enhanced sensitivity in musicianspreviously observed in, e.g., [22], [23], [24]—could not be confirmed.

By considering actively applied forces and unconstrained contact of the finger pad, the present study adopted a somewhat more ecological approach [1] as compared to the studies mentioned above An analogous approach was adopted by Brisben et al. [7], who studied vibrotactile thresholds in an active task that required participants to grab a vibrating cylinder. While the exerted forces were not measured, similar to the results presented here, thresholds were found much lower than what reported in most previous literature: At 150 and $200 \mathrm{~Hz}$ the average displacement threshold was $0.03 \mu \mathrm{m}$ peak (down to $0.01 \mu \mathrm{m}$ in some subjects), which is equivalent to rms acceleration values of $85.5 \mathrm{~dB}$ at $150 \mathrm{~Hz}$, and $90.5 \mathrm{~dB}$ at $200 \mathrm{~Hz}$. The authors suggested that such low figures could be due to the multiple stimulation areas on the hand involved in grabbing the vibrating cylinder, the longitudinal direction of vibration, and the force exerted by the participants. In [12] active movement resulted in lower sensitivity thresholds as compared to passive movement. Other studies also suggest that active touch involving planning or execution of movement results in better percepts [40]. Still in an ecological context, enhanced vibrotactile sensitivity during an active playing task on the piano was reported in a recent study by some of the present authors [15]. The main differences from the present study are found in the presence of auditory cues and the different spectral and temporal nature of the vibrotactile stimuli. Under those conditions, vibrations at the piano keyboard could be clearly perceived (detection rates of up to 90 percent) at amplitudes just close to the thresholds found in the literature for sinusoidal stimuli [16], [17]. In fact, such vibration amplitudes are well above the thresholds presented 
here, confirming the better suitability of our results for predicting vibration perception in active-touch and ecological contexts. Indeed, according to [17], a clear sensation of vibration arises for stimuli $40 \mathrm{~dB}$ above threshold.

\subsection{Measurement Issues}

In general, since different experimental methods may give different perceptual thresholds [6], [38], [39], it is not straightforward to compare results between studies. The one-up-two-down staircase method, adopted here, targets the stimulus level corresponding to 70.7 percent correct responses. However, the estimate approaches the target asymptotically with increasing number of turning points. With a typically low number of reversals per run (eight in our case), the estimate is likely to drift somewhat from the target, even up to about 10 percent, depending on the relative step size [41].

Concerning the characterization of vibration at the touch-box (Section 2.4 and the appendix, available in the online supplemental material), the use of solid test weights and the placement of the measurement accelerometer in parallel to them might have slightly altered the vibration propagation during the validation phase, as compared to the actual case of a pressing finger. A solution could be to repeat measurements with a differently shaped accelerometer, allowing to place weights on top of it, and to simulate the contribution of skin by interposing an equivalently soft material between the test weights and the vibrating panel.

While the overall response of the interface is improved as compared to the preliminary version presented in [21], the spectral centroid of noise vibration was found to generally shift to higher frequencies for higher weights, and, to a lesser extent, to lower frequencies for lower amplitudes. As pointed out in Section 4.1, this might have a slight effect on the measured trend of thresholds for noise vibration.

\section{Conclusion}

An experiment was conducted to study how vibration detection thresholds are affected by active pressing forces. As opposed to most of the existing literature, the experiment did not constrain finger contact area, which showed a nearly logarithmic relationship with pressing force. Significant effects on thresholds were found for both the type of stimuli, which consisted in either a sinusoidal or a broadband vibration, and the force level. In particular, when representing force on a logarithmic scale, $\mathrm{dB}$ thresholds resulted in a decreasing, almost linear trend for sinusoidal stimuli, and a U-shape trend for noise stimuli. Overall, the measured sensitivity thresholds are considerably lower than what previously reported in the literature. We argue that the effects on threshold were likely caused by the combination of the following psychophysical factors: i) enhanced mechanical coupling between the skin, tendons, bones and the vibrating surface, due to pressing force; ii) large contact area, covering up to the whole finger pad, resulting in spatial summation for the Pacinian channel; iii) long duration stimuli $(1.5 \mathrm{~s})$, resulting in temporal summation.
The presented results may be useful to optimize vibrotactile feedback in human-computer interfaces. The ability to predict thresholds according to the measured finger pressing force or contact area would enable the adaptation of vibrotactile feedback amplitude and frequency to the user's gestures. As an example, feedback that is too intense might result in distraction rather than aid, which could be a serious issue for teleoperation in critical environments. From a technological perspective, the reported effect of finger pressing force on sensitivity thresholds implies lower power requirements and lower mechanical strain for vibration actuators. As a general observation, we suggest that studies addressing active touch psychophysics could inform novel guidelines for the design and implementation of haptic feedback.

\section{ACKNOWLEDGMENTS}

Stefano Papetti, Hanna Järveläinen, Sébastien Schiesser, and Martin Fröhlich were supported by project AHMI (AudioHaptic modalities in Musical Interfaces), funded by the Swiss National Science Foundation (SNSF): http://p3.snf. ch/project-150107. Bruno L. Giordano was supported by the Biotechnology and Biological Sciences Research Council (BBSRC; BB/M009742/1).

\section{REFERENCES}

[1] J. J. Gibson, "Observations on active touch," Psychol. Rev., vol. PR69, pp. 477-491, 1962.

[2] S. J. Bolanowski, G. A. Gescheider, R. T. Verrillo, and C. M. Checkosky, "Four channels mediate the mechanical aspects of touch," J. Acoust. Soc. Am., vol. 84, no. 5, pp. 1680-1694, Nov. 1988.

[3] J. C. Craig and C. E. Sherrick, "The role of skin coupling in the determination of vibrotactile spatial summation," Perception Psychophys., vol. PP-6, no. 2, pp. 97-101, Mar. 1969.

[4] P. J. Lamoré and C. J. Keemink, "Evidence for different types of mechanoreceptors from measurements of the psychophysical threshold for vibrations under different stimulation conditions," J. Acoust. Soc. Am., vol. 83, no. 6, pp. 2339-2351, Jun. 1988.

[5] N. Harada and M. J. Griffin, "Factors influencing vibration sense thresholds used to assess occupational exposures to hand transmitted vibration," Br. J. Ind. Med., vol. 48, no. 48, pp. 185-192, Mar. 1991.

[6] S. Maeda and M. J. Griffin, "A comparison of vibrotactile thresholds on the finger obtained with different equipment," Ergonomics, vol. 37, no. 8, pp. 1391-1406, Aug. 1994.

[7] A. J. Brisben, S. S. Hsiao, and K. O. Johnson, "Detection of vibration transmitted through an object grasped in the hand," J. Neurophysiol, vol. 81, no. 4, pp. 1548-1558, Apr. 1999.

[8] R. T. Verrillo, "Psychophysics of vibrotactile stimulation," J. Acoust. Soc. Am., vol. ASA-77, no. 1, pp. 225-232, 1985.

[9] R. T. Verrillo, "Temporal summation in vibrotactile sensitivity," J. Acoust. Soc. Am., vol. ASA-37, pp. 843-846, May 1965.

[10] G. A. Gescheider and J. M. Joelson, "Vibrotactile temporal summation for threshold and suprathreshold levels of stimulation," Perception Psychophys., vol. PP-33, no. 2, pp. 156-162, Mar. 1983.

[11] D. J. Whitehouse and M. J. Griffin, "A comparison of vibrotactile thresholds obtained using different diagnostic equipment: The effect of contact conditions," Int. Arch. Occup. Environ. Health, vol. 75, no. 1/2, pp. 85-89, Jan. 2002.

[12] M. Z. Yildiz, I. Toker, F. B. Özkan, and B. Güçlü, "Effects of passive and active movement on vibrotactile detection thresholds of the Pacinian channel and forward masking," Somatosens. Mot. Res., vol. 32, no. 4, pp. 262-272, Oct. 2015.

[13] G. Békésy, "Über Die Vibrationsempfindung [On the sensation of vibration]," Akust. Z., Leipzig, Germany: Hirzel Verlag, vol. 4, pp. 316-334, 1939.

[14] L. Wyse, S. Nanayakkara, P. Seekings, S. H. Ong, and E. A. Taylor. (2012). [Online]. "Palm-area sensitivity to vibrotactile stimuli above $1 \mathrm{kHz}$, , in Proc. Conf. New Interf. Music. Expr., Available: http://www.nime.org/archives/ 
[15] F. Fontana, F. Avanzini, H. Järveläinen, S. Papetti, F. Zanini, and V. Zanini, "Perception of interactive vibrotactile cues on the acoustic grand and upright piano," in Proc. Int. Conf. Sound Music Comput., 2014, pp. 948-953.

[16] A. Askenfelt and E. V. Jansson, "On vibration sensation and finger touch in stringed instrument playing," Music Perception, vol. 9, no. 3, pp. 311-349, 1992.

[17] R. T. Verrillo, "Vibration sensation in humans," Music Perception, vol. 9, no. 3, pp. 281-302, 1992.

[18] D. Prattichizzo, F. Chinello, C. Pacchierotti, and M. Malvezzi, "Towards wearability in fingertip haptics: A 3-DoF wearable device for cutaneous force feedback," IEEE Trans. Haptics, vol. 6, no. 4, pp. 506-516, Oct. 2013.

[19] O. Bau, I. Poupyrev, A. Israr, and C. Harrison, "TeslaTouch : Electrovibration for touch surfaces," in Proc. 23nd Annu. ACM Symp. User Interface Softw. Technol., 2010, pp. 283-292.

[20] E. C. Chubb, J. E. Colgate, and M. A. Peshkin, "ShiverPaD: A glass haptic surface that produces shear force on a bare finger," IEEE Trans. Haptics, vol. 3, no. 3, pp. 189-198, Jul.-Sep. 2010.

[21] S. Papetti, H. Järveläinen, and G.-M. Schmid, "Vibrotactile sensitivity in active finger pressing," in Proc. IEEE World Haptics Conf., 2015, pp. 457-462.

[22] A. M. Zamorano, I. Riquelme, B. Kleber, E. Altenmüller, S. M. Hatem, and P. Montoya, "Pain sensitivity and tactile spatial acuity are altered in healthy musicians as in chronic pain patients," Frontiers Hum. Neurosci., vol. 8, Jan. 2014, Art. no. 1016.

[23] P. Ragert, A. Schmidt, E. Altenmüller, and H. R. Dinse, "Superior tactile performance and learning in professional pianists: Evidence for meta-plasticity in musicians," Eur. J. Neurosci., vol. 19, no. 2, pp. 473-478, Jan. 2004.

[24] H. R. Dinse, T. Kalisch, P. Ragert, B. Pleger, P. Schwenkreis, and M. Tegenthoff, "Improving human haptic performance in normal and impaired human populations through unattended activation-based learning," ACM Trans. Appl. Perception, vol. 2, no. 2, pp. 71-88, 2005.

[25] M. Civolani, F. Fontana, and S. Papetti, "Efficient acquisition of force data in interactive shoe designs," in Proc. 5th Int. Workshop Haptic Audio Interaction Des., 2010, pp. 129-138.

[26] M. Yamaoka, A. Yamamoto, and T. Higuchi, "Basic analysis of stickiness sensation for tactile displays," in Haptics: Perception, Devices and Scenarios, M. Ferre, Ed. Berlin, Germany: Springer, 2008, vol. 5024, pp. 427-436.

[27] A. DiDomenico Astin, "Finger Force Capability: Measurement and Prediction Using Anthropometric and Myoelectric Measures," M.S. thesis, Virginia Polytechnic Inst. State Univ., Blacksburg, VA, 1999.

[28] H. Levitt, "Transformed Up-Down methods in Psychoacoustics," J. Acoust. Soc. Am., vol. ASA-49, no. 2, pp. 467-477, 1971.

[29] C. M. Salisbury, R. B. Gillespie, H. Z. Tan, F. Barbagli, and J. K. Salisbury, "What you can't feel won't hurt you: Evaluating haptic hardware using a haptic contrast sensitivity function," IEEE Trans. Haptics, vol. 4, no. 2, pp. 134-146, Mar./Apr. 2011

[30] L. Ekenvall, G. Gemne, and R. Tegner, "Correspondence between neurological symptoms and outcome of quantitative sensory testing in the hand-arm vibration syndrome," Brit. J. Ind. Med., vol. 46, pp. 570-574, 1989.

[31] S. Aatola, M. Färkkilä, I. Pyykkö, and O. Korhonen, "Measuring method of vibration perception threshold of fingers and its application to vibration exposed workers," Int. Archives Occupational Environ. Health, vol. 62, pp. 239-242, 1990.

[32] O. Aaserud, J. Juntunen, and E. Matikainen, "Vibration sensitivity thresholds: Methodological considerations," Acta Neurologica Scandinavica, vol. 82, pp. 277-283, 1990.

[33] H.-Y. Jeong, T. Gosho, M. Higashimori, and M. Kaneko, "Frequency response dependence to vibration sensitivity by pressing," in Proc. IEEE Int. Conf. Robot. Biomimetics., Feb. 2008, pp. 1206-1211.

[34] G. A. Gescheider, S. J. Bolanowski, R. T. Verrillo, D. J. Arpajian, and T. F. Ryan, "Vibrotactile intensity discrimination measured by three methods," I. Acoust. Soc. Am., vol. 87, no. 1, Jan. 1990, Art. no. 330

[35] C. Wells, L. M. Ward, R. Chua, and J. Timothy Inglis, "Touch noise increases vibrotactile sensitivity in old and young," Psychol. Sci., vol. 16, no. 4, pp. 313-320, Apr. 2005

[36] G. W. Young, D. Murphy, and J. Weeter, "Auditory discrimination of pure and complex waveforms combined with vibrotactile feedback," presented at the New Interfaces Music. Expr., Baton Rouge, LA, USA, 2015.
[37] G. Gescheider, S. Bolanowski, and R. Verrillo, "Some characteristics of tactile channels," Behav. Brain Res., vol. 148, no. 1/2, pp. 3540, Jan. 2004.

[38] M. Morioka and M. J. Griffin, "Dependence of vibrotactile thresholds on the psychophysical measurement method," Int. Arch. Occup. Environ. Health, vol. 75, no. 1/2, pp. 78-84, Jan. 2002.

[39] B. Harazin, J. Kuprowski, and G. Stolorz, "Repeatability of vibrotactile perception thresholds obtained with two different measuring systems," Int. J. Occup. Med. Environ. Health, vol. 16, no. 4, pp. 311-319, 2003.

[40] G. H. Van Doorn, V. Dubaj, D. B. Wuillemin, B. L. Richardson, and M. A. Symmons, "Cognitive Load Can Explain Differences in Active and Passive Touch," in Haptics Perception, Devices, Mobility, Commun., P. Isokoski and J. Springare, Eds. Berlin, Germany: Springer, 2012, vol. 7282, pp. 91-102.

[41] M. A. Garcia-Perez, "Forced-choice staircases with fixed step sizes: Asymptotic and small-sample properties," Vis. Res., vol. 38, pp. 1861-1881, 1998

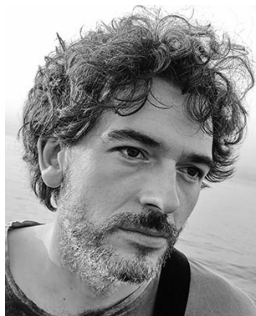

Stefano Papetti received the Laurea (MSc) degree in computer engineering from the University of Padova, Italy, in 2006, and the PhD degree in computer science from the University of Verona, Italy, in 2010. From 2006 to 2011, he was a research associate in the Computer Science Department, University of Verona. Since 2011, he has been a research associate in the Institute for Computer Music and Sound Technology, Zurich University of the Arts, Switzerland, where he led a research project on haptic musical interfaces funded by the Swiss National Science Foundation (SNSF). His research activity focuses on the design and evaluation of auditory and haptic interfaces, and on models and applications for interactive sound synthesis.

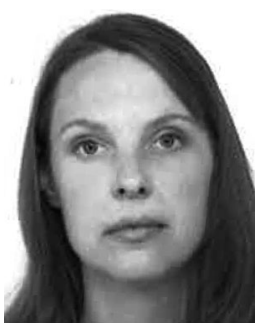

Hanna Järveläinen received the $\mathrm{MSc}$ and $\mathrm{PhD}$ degrees in electronic and communications engineering from the Helsinki University of Technology (HUT, now Aalto University), Finland, in 1997 and 2003, respectively, and the bachelor's of music degree in music theory and composition from the Sibelius Academy of the Helsinki University of the Arts, Finland, in 2006. She was a research scientist at the HUT Laboratory of Acoustics and audio signal processing until 2006 and has worked as a research associate in the Institute for Computer Music and Sound Technology, Zurich University of the Arts, Switzerland, where her research topics include multimodal perception of music and musical gesture since 2012. She spent the wild years between her proper academic jobs singing early music.

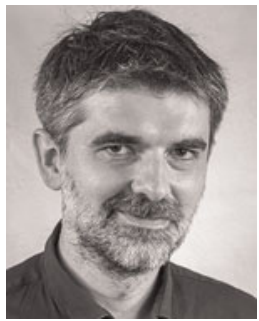

Bruno L. Giordano received the Laurea (Msc) degree in experimental psychology from the University of Padova, Italy, in 2001. In 2006, he received the $\mathrm{PhD}$ degree in perception and psychophysics from the Department of General Psychology, University of Padova, jointly supervised by STMS-IRCAM-CNRS, Paris, France. From 2006-2011, he carried out research on the psychophysics of natural sounds and of audio-haptic integration in the Department of Music Research, McGill University, Montréal, Canada. He is currently a research associate in the Institute of Neuroscience and Psychology of the University of Glasgow, Scotland, where he has been carrying out research on the functional anatomy and cortical dynamics of auditory processes since 2011. 


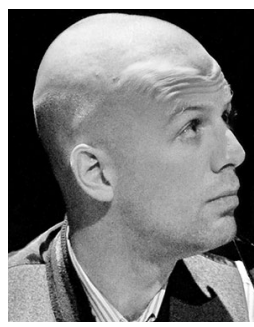

Sébastien Schiesser received the MSc degree in microtechnology from the Swiss Federal Institute of Technology (EPFL), followed by a performance degree (saxophone) from the Zurich University of the Arts. Currently, he works both as a freelance musician and as a research associate in the Institute for Computer Music and Sound Technology, Zurich University of the Arts, Switzerland, where he designs electronics and develops new musical interfaces.

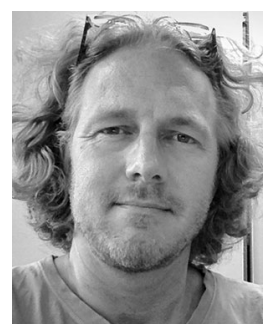

Martin Fröhlich received the degrees in mechanical engineering and media art. He has been working for 10 years in a variety of software companies around the world. Today, in parallel with his artistic activity, he works in the Institute for Computer Music and Sound Technology, Zurich University of the Arts, Switzerland, where he mainly develops multimodal interfaces.

$\triangleright$ For more information on this or any other computing topic, please visit our Digital Library at www.computer.org/publications/dlib. 